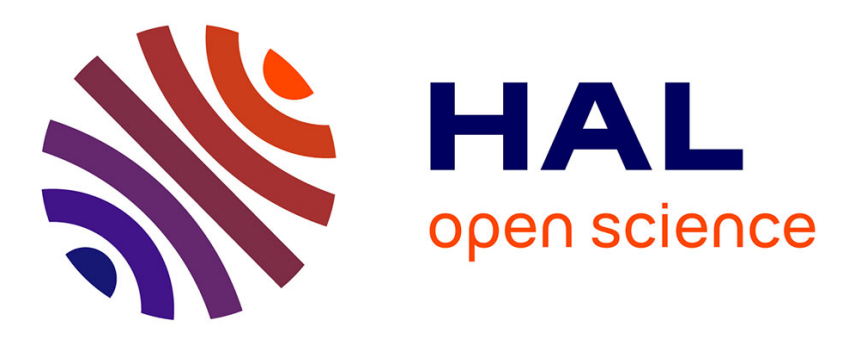

\title{
Flux de matières en suspension des fleuves côtiers se jetant dans l'Etang de Berre (Bouches-du-Rhône, France)
}

\author{
Mirabelle Fiandino, Claude Martin
}

\section{- To cite this version:}

Mirabelle Fiandino, Claude Martin. Flux de matières en suspension des fleuves côtiers se jetant dans l'Etang de Berre (Bouches-du-Rhône, France). La Houille Blanche - Revue internationale de l'eau, 2004, 2004 (4), pp.97-1001. hal-00315666

\section{HAL Id: hal-00315666 \\ https://hal.science/hal-00315666}

Submitted on 29 Aug 2008

HAL is a multi-disciplinary open access archive for the deposit and dissemination of scientific research documents, whether they are published or not. The documents may come from teaching and research institutions in France or abroad, or from public or private research centers.
L'archive ouverte pluridisciplinaire HAL, est destinée au dépôt et à la diffusion de documents scientifiques de niveau recherche, publiés ou non, émanant des établissements d'enseignement et de recherche français ou étrangers, des laboratoires publics ou privés. 


\title{
Flux de matières en suspension des fleuves côtiers se jetant dans l'Étang de Berre (Bouches-du-Rhône, France)
}

\author{
Fluxes of suspended matter in the coastal rivers towards \\ the Pond of Berre (Bouches-du-Rhône, France)
}

\author{
Mirabelle Fiandino*, Claude Martin** \\ * 13 Rue des Jasses, F-13510 Eguilles, France \\ E-mail : mfiandin@club-internet.fr \\ **UMR 6012 "ESPACE", Département de Géographie, 98 Boulevard Edouard Herriot, BP 3209, \\ F-06204 Nice Cedex 03, France \\ E-mail : martincl@infonie.fr
}

\begin{abstract}
Résumé
L'Etang de Berre est un vaste bassin d'eau saumâtre en communication avec la Méditerranée. Il sert d'exutoire à trois principaux cours d'eau à régime méditerranéen marqué: l'Arc, la Touloubre et la Cadière. Depuis plusieurs décennies, l'Etang de Berre connaît un profond dysfonctionnement, du fait d'une forte anthropisation de son bassin versant et, à partir de 1966, des apports d'eau douce et de sédiments en provenance de la Durance par le canal EDF. La présente étude propose une quantification des flux de MES, provenant de l'Arc de la Touloubre et de la Cadière, de septembre 1998 à décembre 2000 et l'extension dans le temps des résultats obtenus (période 1961-2000). Sur la période 1961-2000, les valeurs calculées des exportations spécifiques annuelles moyennes de MES atteignent 35,5 t/ $\mathrm{km}^{2}$ pour l'Arc, 17,4 t/ $\mathrm{km}^{2}$ pour la Touloubre et 47,7 t/ $\mathrm{km}^{2}$ pour la Cadière. Près de 30 \% des MES arrivant dans l'Etang de Berre proviennent actuellement de son bassin versant.
\end{abstract}

\begin{abstract}
The Pond of Berre is a large briny water pond in communication with the Mediterranean sea. It is a discharge system of three main rivers presenting a marked Mediterranean flow: the Arc, the Touloubre and the Cadière. For several decades, the Pond of Berre undergoes a serious dysfonction due to the high level of the anthropic activities of the catchment area and, since 1966, to contributions of fresh water and sediments coming from the Durance by EDF channel. The present study proposes the quantification of fluxes of suspended matter, coming from the Arc, the Touloubre and the Cadiere, from September 1998 to December 2000 and the extension in the time of the obtained results (period 1961-2000). Over the period 1961-2000, the average annual specific computed values of suspended matter are $35,5 \mathrm{t} / \mathrm{km}^{2}$ for the Arc, $17,4 \mathrm{t} / \mathrm{km}^{2}$ for the Touloubre and $47,7 \mathrm{t} / \mathrm{km}^{2}$ for the Cadière. Nearly $30 \%$ as of the suspended matter carried towards the Pond of Berre, originate actually on its catchment area.
\end{abstract}

\section{Iø INTRODUCTION}

Situé dans le Sud-Est de la France, l'Etang de Berre est un vaste bassin d'eau saumâtre en communication avec la Méditerranée. Exutoire de trois principaux cours d'eau à régime méditerranéen marqué, l'Arc, la Touloubre et la Cadière (fig. 1), il constitue un milieu côtier fragile. Depuis plusieurs décennies, l'Etang de Berre connaît des modifications de son fonctionnement "naturel", du fait d'une forte anthropisation de son bassin versant, et des apports d'eau douce et de sédiments en provenance de la Durance par le canal usinier EDF, qui sont venus s'ajouter à ceux des fleuves, à partir de 1966. Le présent article est consacré à la quantification des exportations de matières en suspension par l'Arc, la Touloubre et la Cadière [1]. Il ne s'intéresse donc pas à la quantification des flux du canal usinier, qui sont contrôlés par EDF, et dont nous est seulement connu le tonnage annuel maximum autorisé. 


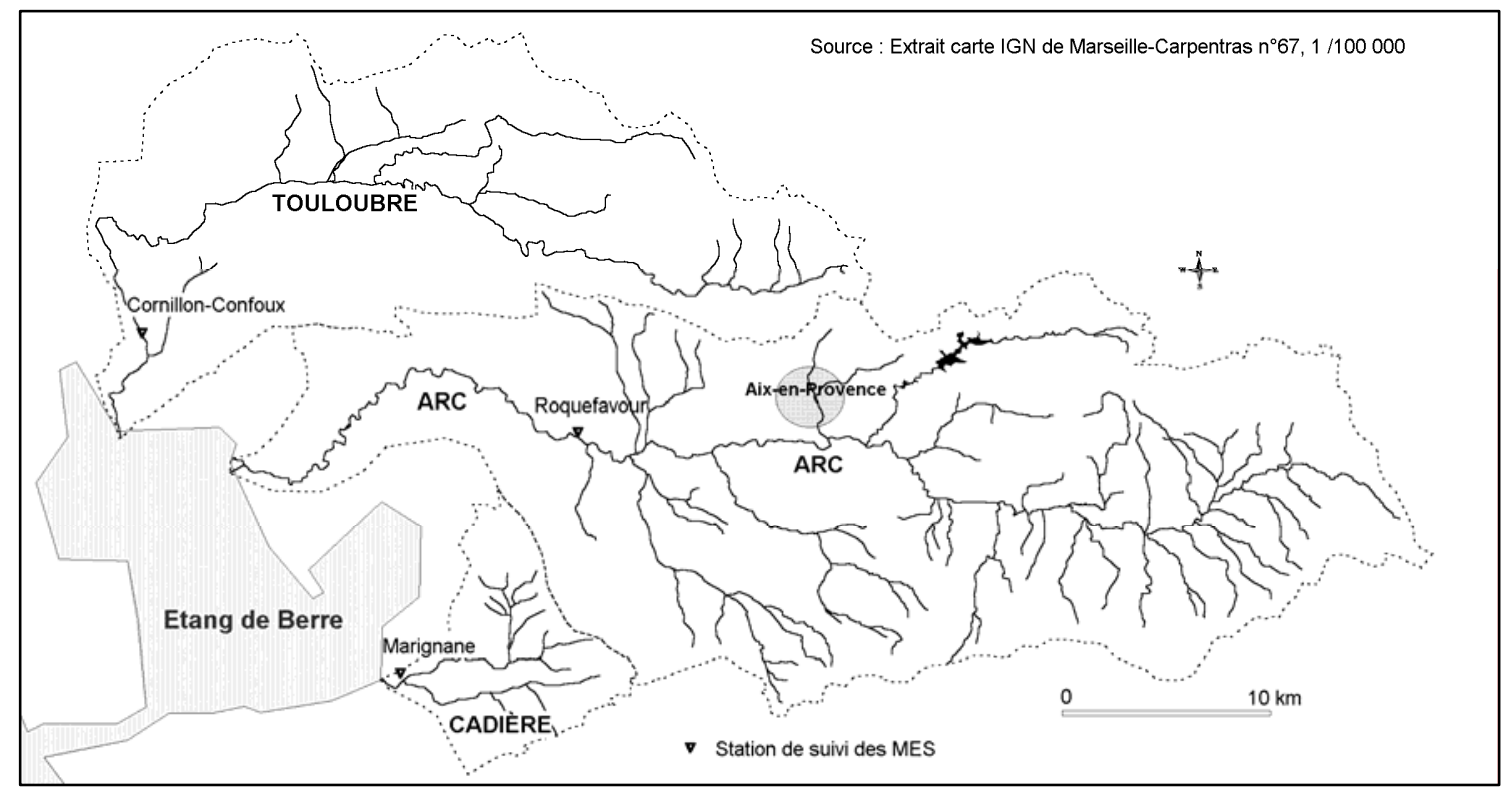

Figure 1 - Présentation du bassin versant de l'Etang de Berre.

\section{IIEDESCRIPTION DU BASSIN VERSANT DE L'ÉTANG DE BERRE}

\section{II.1 Caractères du bassin versant}

Les bassins de l'Arc $\left(720 \mathrm{~km}^{2}\right)$, de la Touloubre $\left(400 \mathrm{~km}^{2}\right)$ et de la Cadière $\left(73 \mathrm{~km}^{2}\right)$ couvrent au total une superficie de $1193 \mathrm{~km}^{2}$, soit $88 \%$ du bassin versant total de l'Etang de Berre. Ils associent des reliefs constitués de roches calcaires (altitudes maximales: $1011 \mathrm{~m}$ dans le massif de la Sainte-Victoire pour le bassin de l'Arc ; $501 \mathrm{~m}$ pour le bassin de la Touloubre ; $272 \mathrm{~m}$ pour celui de la Cadière) à des plaines alluviales dégagées dans des roches tendres (argiles, marnes et/ou molasse). Ces bassins diffèrent sensiblement par l'occupation du sol (tab. I).

Tableau I - Densité de population et occupation du sol dans les bassins versants.

\begin{tabular}{|c|c|c|c|}
\hline & Arc & Touloubre & Cadière \\
\hline Densité de population & 350 habitants $/ \mathrm{km}^{2}$ & 250 habitants $/ \mathrm{km}^{2}$ & 1400 habitants $/ \mathrm{km}^{2}$ \\
\hline Occupation du sol & Agriculture : 40 & Agriculture : 53 & Agriculture : 25 \\
\hline (en \% de la superficie & Pinèdes, garrigues : 50 & Pinèdes, garrigues : 42,5 & Pinèdes, garrigues : 50 \\
\hline totale) & Urbanisation : 10 & Urbanisation : 4,5 & Urbanisation : 25 \\
\hline
\end{tabular}

\section{II.2 Les aspects climatiques et hydrologiques}

Les bassins de l'Arc, de la Touloubre et de la Cadière sont soumis à un climat de type méditerranéen présentant des précipitations généralement abondantes en automne et une période sèche en été. Sur la période 1961-2000, les précipitations annuelles moyennes au cœur du terrain d'étude, à la station d'Aix-Galice, s'établissent à $616 \mathrm{~mm}$ (écart type : $150 \mathrm{~mm}$ ). Toutefois les précipitations décroissent globalement d'est en ouest, les valeurs annuelles moyennes passant de plus de $800 \mathrm{~mm}$ à l'extrémité orientale du bassin de l'Arc, à moins de 550 mm sur le bassin de la Cadière. 
Les pluies peuvent être très intenses ( $77 \mathrm{~mm}$ en une heure le 7 septembre 1998 sur le bassin de la Cadière ; 53 mm en une heure le 19 septembre 2000 sur le bassin de l'Arc). Elles peuvent être la cause de ruissellements importants, générateurs d'érosion.

Les débits spécifiques annuels moyens disponibles sont de $4,5 \mathrm{l} / \mathrm{s} / \mathrm{km}^{2}$ pour l'Arc à Saint-Pons (605 $\mathrm{km}^{2}$ - période 1975-1995), de $8,2 \mathrm{l} / \mathrm{s} / \mathrm{km}^{2}$ pour la Touloubre à Saint-Chamas (392 km² - période 1991-1997) et de $11,6 \mathrm{l} / \mathrm{s} / \mathrm{km}^{2}$ pour la Cadière à Marignane $\left(72,4 \mathrm{~km}^{2}\right.$ - période 1984-2000). Sous la dépendance des conditions pluviométriques, l'Arc et la Cadière sont soumis à un régime hydrologique marqué par une période d'abondance en hiver, et par une période de basses eaux en été. Au contraire, la Touloubre connaît une longue période d'abondance hydrologique du printemps à l'automne, en relation avec les rejets des canaux d'irrigation alimentés par des eaux de la Durance, et une courte période de basses eaux en hiver. Le bassin de la Cadière est également soumis à des entrées d'eau extérieures au bassin, soit par des sources karstiques, soit par l'intermédiaire du canal de Marseille.

A l'échelle journalière, les trois cours d'eau sont soumis à des crues particulièrement fortes en automne et en hiver. Les débits journaliers spécifiques ont atteint $372 \mathrm{l} / \mathrm{s} / \mathrm{km}^{2}$ pour l'Arc en janvier 1978, 308 l/s/ $/ \mathrm{km}^{2}$ pour la Cadière en septembre 1993 et 68 l/s/km² pour la Touloubre en février 1994.

\section{IIInMÉTHODOLOGIE}

\section{III.1 Protocole de mesure des flux de matières en suspension}

Afin de mener cette étude, un préleveur automatique d'échantillons d'eau a été implanté sur chaque cours d'eau, au niveau d'une station hydrométrique située dans sa partie aval, à Roquefavour pour l'Arc (bassin de $650 \mathrm{~km}^{2}$ ), à Cornillon-Confoux pour la Touloubre (bassin de $386 \mathrm{~km}^{2}$ ) et à Marignane pour la Cadière. De nombreuses séries d'échantillons ont ainsi été collectées lors des crues et en basses eaux. Les campagnes de prélèvements automatiques ont débuté en septembre 1998 sur la Cadière et la Touloubre et en février 1999 sur l'Arc, et se sont terminées en décembre 2000. Les crues ont été particulièrement étudiées, car elles assurent la plus grande partie des transports de MES. Toutefois les périodes de basses eaux n'ont pas été négligées. Les campagnes de prélèvements automatiques ont été complétées par des prélèvements manuels lors des passages sur le terrain.

Au cours de la période des mesures, une centaine d'événements pluviométriques se sont produits, parmi lesquels une trentaine ont engendré des crues plus ou moins fortes et se sont révélés efficaces du point de vue hydrosédimentaire. Pour l'Arc, nous avons réalisé 25 campagnes lors des crues et 10 en basses eaux. Pour la Touloubre, 18 campagnes ont été réalisées lors des crues et 16 en basses eaux. Une vingtaine de campagnes ont été effectuées pour la Cadière lors des crues et 11 en basses eaux. Les préleveurs automatiques ont été programmés sur un pas de temps d'une heure ou d'une heure et demie pour la Cadière, et de deux heures pour l'Arc et la Touloubre.

La turbidité spécifique a été déterminée sur 2500 échantillons d'eau, par pesée des matières en suspension après décantation, vidange de l'eau et séchage à $40^{\circ} \mathrm{C}$. La part de la matière organique a été mesurée sur 160 échantillons, en calcinant les matières en suspension à $500^{\circ} \mathrm{C}$.

Des prélèvements manuels en différents points de la section transversale des cours d'eau, ont montré que les préleveurs automatiques fournissaient une estimation de la turbidité spécifique des eaux écoulées avec un risque d'erreur de l'ordre de $5 \%$. 


\section{III.2 Calcul des flux lors des campagnes de mesure des MES}

Pour les épisodes de crue, les flux de particules en suspension sont déterminés à pas de temps variable [2], en prenant en compte tous les points de l'hydrogramme utilisés pour évaluer les écoulements. En effet, pour chaque épisode hydrologique, il est possible de déterminer une équation de régression permettant de calculer la concentration des MES à partir du débit [3] [4]. Pour chaque point de l'hydrogramme, le flux instantané est donc finalement obtenu en multipliant le débit par la concentration calculée des MES.

En basses eaux, les relations entre les concentrations des MES et les débits ne sont pas très fortes. Dans ces conditions, les flux journaliers de MES ont été estimés en utilisant la moyenne des concentrations des MES mesurées au cours de la journée.

\section{III.3 Extension dans le temps des mesures des flux}

Les flux sédimentaires mesurés ont été corrélés, d'une part, aux données hydrologiques et, d'autre part, aux données pluviométriques.

\section{III.3.1 Relations entre les flux de MES et les conditions hydrologiques}

Des équations de régression liant les flux de MES aux conditions hydrologiques ont été établies en corrélant les flux sédimentaires journaliers mesurés aux débits journaliers, pour les basses eaux, ou à un indice (Iq), pour les crues.

Dans le cas des crues, l'indice Iq indique l'efficacité potentielle des phénomènes érosifs en fonction des écoulements. Etabli de manière empirique, il est de la forme :

$\mathrm{Iq}=\mathrm{a} \mathrm{Qj}+\mathrm{b}$ Qimax

avec Qj : débit journalier en $\mathrm{m}^{3} / \mathrm{s}$ et Qimax : débit maximum instantané en $\mathrm{m}^{3} / \mathrm{s}$, sachant que $\mathrm{a}+\mathrm{b}=1$.

\section{III.3.2 Relations entre les flux de MES et les conditions pluviométriques}

Les régressions ont été étudiées sur la période d'observation des flux de MES, mais en travaillant à l'échelle mensuelle. Les flux mensuels ont été évalués en complétant les mesures effectuées lors des épisodes ayant donné lieu à un suivi, par une reconstitution des valeurs journalières fondée sur les relations flux - débits (voir supra) pour les périodes sans suivi. Les flux mensuels de MES ainsi déterminés sont corrélés aux pluies journalières maximales des mois correspondants, à travers un indice (Ip) représentant l'agressivité potentielle des pluies au cours du mois :

Ip = a Pmj + b Pnj

avec Pmj et Pnj : pluies maximales en $m$ et $n$ jours au cours du mois.

\section{IVaRÉSULTATS ET INTERPRÉTATION}

\section{IV.1 Les campagnes de mesure des MES}

Au cours de la période de mesure des MES, l'Arc et la Cadière ont présenté les plus fortes crues (débits instantanés de l'ordre de $100 \mathrm{~m}^{3} / \mathrm{s}$ pour l'Arc et plus de $50 \mathrm{~m}^{3} / \mathrm{s}$ pour la Cadière) et les plus fortes concentrations des MES ( $4 \mathrm{~g} / \mathrm{l}$ pour l'Arc et 3,5 g/l pour la Cadière), alors que pour la Touloubre les débits instantanés maximaux n'ont pas dépassé 15 m³/s et les concentrations des MES 2,2 g/l.

Les concentrations moyennes des MES en basses eaux avoisinent $0,05 \mathrm{~g} / \mathrm{l}$ pour la Cadière ; $0,06 \mathrm{~g} / \mathrm{l}$ pour la Touloubre ; et $0,03 \mathrm{~g} / \mathrm{l}$ pour l'Arc. Elles sont dues aux rejets des stations d'épuration et aux rejets domestiques directs, et pour la Touloubre aux rejets des canaux d'irrigation. 
Pour les trois cours d'eau, la majeure partie des MES n'en sont pas moins transportées pendant les crues [5]. Ainsi l'Arc a-t-il évacué plus de 8000 tonnes de MES en 72 heures lors de l'épisode du 17 au 19 janvier 1999 (suivi par des prélèvements manuels), ce qui correspond à un taux d'érosion spécifique de $12,3 \mathrm{t} / \mathrm{km}^{2}$. Au cours du même épisode, les exportations spécifiques de la Cadière ont été de $5,7 \mathrm{t} / \mathrm{km}^{2}$ en 48 heures, et celles de la Touloubre de $0,25 \mathrm{t} / \mathrm{km}^{2}$ en 40 heures. Les différences entre les bassins versants s'expliquent par les conditions pluviométriques: des précipitations journalières abondantes, supérieures à 60 et même à $75 \mathrm{~mm}$, ont été mesurées le 17 janvier sur le bassin de l'Arc, alors que les pluies ont atteint moins de $50 \mathrm{~mm}$ sur celui de la Cadière et à peine $40 \mathrm{~mm}$ sur une bonne partie du bassin de la Touloubre.

Lors des campagnes de prélèvements en crue, les taux de matière organique dans les MES ont été compris entre 5 et $15 \%$ pour l'Arc ( 4 campagnes), 7 et $15 \%$ pour la Touloubre ( 3 campagnes) et 15 et $30 \%$ pour la Cadière ( 3 campagnes). En basses eaux, les eaux ont présenté des teneurs en matière organique de 10 à $29 \%$ pour l'Arc (2 campagnes de 1 et 2 jours), de $6 \%$ environ pour la Touloubre (2 campagnes de 3 jours chacune) et de $46 \%$ environ pour la Cadière (une seule campagne d'une journée).

\section{IV.1.1 Bilans des flux de MES}

Lois générales de fonctionnement hydrosédimentaire d'après les conditions hydrologiques

Les corrélations entre les flux sédimentaires mesurés et les paramètres hydrologiques sont assez bonnes et se révèlent significatives avec un risque d'erreur de $5 \%$. Pour les trois cours d'eau, les débits expliquent plus de $90 \%$ des flux de MES en période de crue et plus de $60 \%$ lors des faibles écoulements (tab. II).

Tableau II - Lois générales de fonctionnement hydrosédimentaire des cours d'eau d'après les conditions hydrologiques.

\begin{tabular}{|l|l|l|}
\hline \multicolumn{1}{|c|}{ Bassin } & \multicolumn{1}{|c|}{ Crues } & \multicolumn{1}{c|}{ Faibles écoulements } \\
\hline Arc & $\begin{array}{l}\text { Fj }=0,79560 \mathrm{Iq}^{2}+123,09729 \mathrm{Iq}-733,10436 \\
\mathrm{r}=0,95 \text { et }^{2}=0,91, \\
\text { avec Iq }=(0,8 \mathrm{Qj}+0,2 \text { Qimax) (DQ/Qimax) }\end{array}$ & $\begin{array}{l}\mathrm{Fj}=9,5315 \mathrm{Qj}-9,0776 \\
\mathrm{r}=0,82 \text { et } \mathrm{r}^{2}=0,68\end{array}$ \\
\hline Touloubre & $\begin{array}{l}\text { Fj }=1,2249 \mathrm{Iq}^{3}-10,574 \mathrm{I} \mathrm{Iq}^{2}+56,074 \mathrm{Iq}-81,957 \\
\mathrm{r}=0,95 \text { et } \mathrm{r}^{2}=0,90, \\
\text { avec Iq } 0,7 \mathrm{Qj}+0,3 \text { Qimax }\end{array}$ & $\begin{array}{l}\text { Fj }=8,2907 \mathrm{Qj}-8,5894 \\
\mathrm{r}=0,77 \text { et } \mathrm{r}^{2}=0,60\end{array}$ \\
\hline Cadière & $\begin{array}{l}\text { Fj }=0,1795 \mathrm{Iq}^{3}-3,1021 \mathrm{Iq}^{2}+46,7 \mathrm{Iq}-45,313 \\
\mathrm{r}=0,98 \text { et } \mathrm{r}^{2}=0,97,\end{array}$ & $\begin{array}{l}\mathrm{Fj}=3,9371 \mathrm{Qj} \\
\mathrm{r}=0,91 \text { et } \mathrm{r}^{2}=0,82\end{array}$ \\
\hline
\end{tabular}

Fj : Flux journalier de MES (tonnes). Iq : Indice traduisant l'agressivité érosive journalière potentielle. $\mathrm{r}$ : Coefficient de corrélation. $\mathrm{r}^{2}$ : Coefficient de détermination. Qj : Débit journalier, en $\mathrm{m}^{3} / \mathrm{s}$. Qimax : Débit instantané maximum, en $\mathrm{m}^{3} / \mathrm{s}$. DQ : Différence entre les débits instantanés maximum et minimum, en $\mathrm{m}^{3} / \mathrm{s}$.

Lois générales de fonctionnement hydrosédimentaire d'après les conditions pluviométriques

Les corrélations entre les flux mensuels de MES et les paramètres pluviométriques sont également assez bonnes et significatives, avec un risque d'erreur de $5 \%$ (tab. III). 
Tableau III - Lois générales de fonctionnement des cours d'eau d'après les conditions pluviométriques.

\begin{tabular}{|l|l|c|c|}
\hline \multicolumn{1}{|c|}{ Bassin } & \multicolumn{1}{|c|}{ Equation } & $\begin{array}{c}\text { Coefficient de } \\
\text { corrélation } r\end{array}$ & $\begin{array}{c}\text { Coefficient de } \\
\text { détermination } \mathrm{r}^{2}\end{array}$ \\
\hline Arc & $\begin{array}{l}\text { F/mois }=0,413 \mathrm{Ip}^{2}+19,78 \mathrm{Ip}, \\
\text { avec Ip }=(0,2 \mathrm{P} 2 \mathrm{jc})+(0,8 \mathrm{P} 4 \mathrm{j})\end{array}$ & 0,80 & 0,64 \\
\hline Touloubre & $\mathrm{F} / \mathrm{mois}=10,284 \mathrm{P} 2 \mathrm{jc}+284,74$ & 0,64 & 0,41 \\
\hline Cadière & $\begin{array}{l}\mathrm{F} / \mathrm{mois}=0,0013 \mathrm{Ip}^{3}-0,1658 \mathrm{Ip}^{2}+12,667 \mathrm{Ip}, \\
\text { avec Ip }=(0,6 \mathrm{P} 2 \mathrm{jc}+0,4 \mathrm{P} 4 \mathrm{j})\end{array}$ & 0,96 & 0,92 \\
\hline
\end{tabular}

F/mois : Flux mensuel de MES, en tonnes. Ip : Indice traduisant l'agressivité érosive mensuelle potentielle. P2jc : Pluies maximales en deux jours consécutifs au cours du mois, en mm. P4j : Pluies maximales en quatre jours non consécutifs au cours du mois, en mm.

\section{IV.2 L'extension dans le temps des mesures de flux}

Sur la période janvier 1998 - décembre 2000, les deux méthodes utilisées pour la reconstitution des flux de MES donnent des résultats très voisins (tab. IV). Certes, la période considérée correspond pratiquement à celle des mesures des transports de MES qui ont servi à établir les équations. Mais des informations disponibles pour le bassin de la Cadière confirment la convergence des deux méthodes : sur la période 1984-2000, les flux estimés d'après les débits s'établissent à 58619 tonnes et ceux évalués d'après les précipitations à 54699 tonnes (différence de l'ordre de 7 \%).

Tableau IV - Erosions spécifiques annuelles moyennes sur la période 1998-2000 : comparaison des deux méthodes de calcul.

\begin{tabular}{|l|c|c|c|}
\hline & Arc & Touloubre & Cadière \\
\hline Précipitations annuelles moyennes $(\mathrm{mm})$ & 614 & 582 & 589 \\
\hline Lame d'eau écoulée annuelle moyenne $(\mathrm{mm})$ & 127 & 196 & 340 \\
\hline $\begin{array}{l}\text { Erosion spécifique }\left(\mathrm{t} / \mathrm{km}^{2} / \mathrm{an}\right) \\
\text { d'après les conditions hydrologiques }\end{array}$ & 36,2 & 16,8 & 56,9 \\
\hline $\begin{array}{l}\text { Erosion spécifique }\left(\mathrm{t} / \mathrm{km}^{2} / \mathrm{an}\right) \\
\text { d'après les conditions pluviométriques }\end{array}$ & 38,3 & 17,4 & 56,5 \\
\hline
\end{tabular}

Sur la période 1998-2000, le bassin de la Cadière, qui a subi un épisode orageux particulièrement violent en septembre 1998 (146 mm en 24 heures), présente le plus fort taux d'érosion spécifique annuel moyen avec 56,9 t/ $\mathrm{km}^{2}$. Les bassins de l'Arc et de la Touloubre, quant à eux, ont rencontré des conditions pluviométriques moins agressives, voire même déficientes dans le cas de la Touloubre. Les taux d'érosion spécifiques avoisinent $37 \mathrm{t} / \mathrm{km}^{2} /$ an pour l'Arc et $17 \mathrm{t} / \mathrm{km}^{2} /$ an pour la Touloubre.

L'extension des données fondée sur les conditions pluviométriques, fournit, pour la période 1961-2000, commune aux trois bassins versants, des taux d'érosion spécifiques de 35,5 t/ $\mathrm{km}^{2} / \mathrm{an}$ pour l'Arc (écart type : 14,4 t/ $\mathrm{km}^{2} / \mathrm{an}$ - fig. 2), 17,4 t/ $\mathrm{km}^{2} / \mathrm{an}$ pour la Touloubre (écart type : 2,3 t/ $\mathrm{km}^{2} / \mathrm{an}$ ) et $47,7 \mathrm{t} / \mathrm{km}^{2} /$ an pour la Cadière (écart type : 22,7 t/ $\mathrm{km}^{2} / \mathrm{an}$ ). Ces valeurs reconstituées sont représentatives à la fois des conditions pluviométriques pendant la période étendue et de certains caractères des bassins versants sur la période de mesure des flux (occupation du sol, aménagements, formes d'érosion préexistantes en cours d'évolution). 


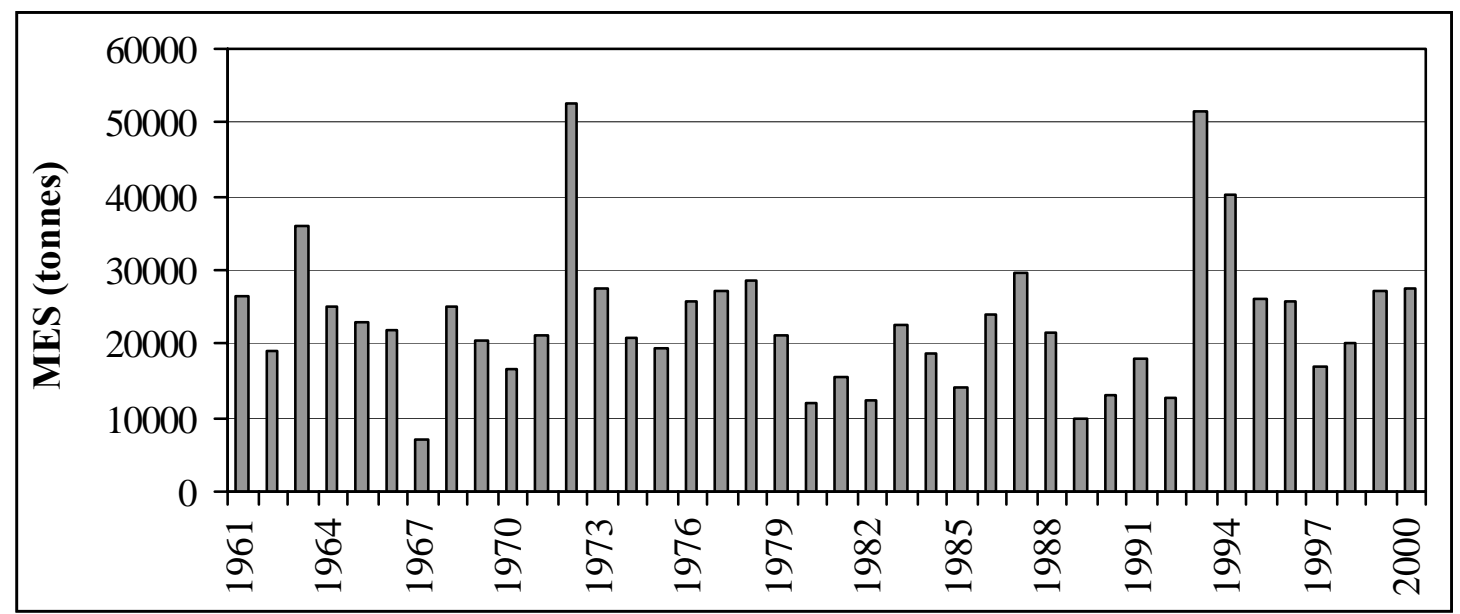

Figure 2 - Exportations annuelles de MES de l'Arc à Roquefavour sur la période 1961-2000.

De 50 à $80 \%$ des exportations de MES de l'Arc et de la Cadière se produisent chaque année au cours des épisodes de crue (sur 25 à $35 \%$ des jours). La Touloubre manifeste un fonctionnement hydrosédimentaire différent. En effet, seulement 30 à $50 \%$ des flux annuels de MES sont liés aux crues.

L'extension dans le temps a pu être menée sur des périodes plus longues pour la Touloubre (1948-2000) et la Cadière (1946-2000) : les résultats (respectivement 14,4 et 46,1 t/ $\mathrm{km}^{2} / \mathrm{an}$ ) sont semblables à ceux obtenus sur la période 1961-2000.

\section{VaCONCLUSION}

Les flux de MES annuels moyens évalués à partir des données pluviométriques de la période 1961-2000, s'établissent à 23102 tonnes pour l'Arc à Roquefavour, 6715 tonnes pour la Touloubre à Cornillon-Corfoux et 3456 tonnes pour la Cadière à Marignane. La superficie contrôlée par les stations de mesure $\left(1108 \mathrm{~km}^{2}\right)$ représente $81 \%$ de la surface totale du bassin versant de l'Etang de Berre $\left(1365 \mathrm{~km}^{2}\right)$. En appliquant aux $257 \mathrm{~km}^{2}$ non contrôlés, les taux d'érosion annuels moyens le plus faible $\left(17,4 \mathrm{t} / \mathrm{km}^{2}\right)$ et le plus fort $\left(47,7 \mathrm{t} / \mathrm{km}^{2}\right)$ trouvés pour les trois bassins versants étudiés, les apports annuels moyens de MES à l'Etang de Berre apparaissent compris entre 37730 et 45520 tonnes. Les fleuves sont donc actuellement responsables de 27 à $31 \%$ des entrées de MES dans l'Etang de Berre, 100000 tonnes étant fournies chaque année par le canal EDF qui amène des eaux de la Durance.

Remerciements : Nous sommes reconnaissants de leur aide à l'ANRT, à la Société Safege-Cetiis, à l'Agence de l'Eau RMC, à EDF, aux Syndicats de l'Arc, de la Touloubre et de la Cadière, au Conseil Général des Bouches-du-Rhône, au Conseil Régional, à la DIREN, aux Services Maritimes et au GIP pour la réhabilitation de l'Etang de Berre.

\section{BIBLIOGRAPHIE}

[1] Fiandino M. (2003). - Apports de matières en suspension par les fleuves côtiers à l'Etang de Berre (Bouches-du-Rhône, France) : quantification des flux, identification des zones sources, étude des relations avec les conditions hydrologiques. Thèse de l'Université de Provence, $325 \mathrm{p}$. (supplément au n XXXI, 2004, des Etudes de Géographie Physique, 298 p., sous presse).

[2] Martin C. (1987). - Les mesures de l'érosion chimique dans les bassins versants de roches cristallines ; comparaison des résultats obtenus par différentes méthodes d'investigation dans le massif des Maures (Var, France), Z. Geomorph., N.F., vol. 31, n 1, p. 73-84.

[3] Bravard J.P. et Petit F. (1997). - Les cours d'eau. Dynamique du système fluvial. Editions Armand Colin, Paris, 222 p. 
[4] Corbonnois J. (1998). - Les facteurs de la variation spatio-temporelle des transports solides et dissous. Géomorphologie, n 4, p. 313-329.

[5] Meybeck M., Pasco A. et Ragu A. (1984). - Evaluation des flux de polluants dans les rivières : Pourquoi, comment et à quel prix ? Collection des Cahiers Techniques Inter Agence, $n^{\circ} 28,23 \mathrm{p}$. 


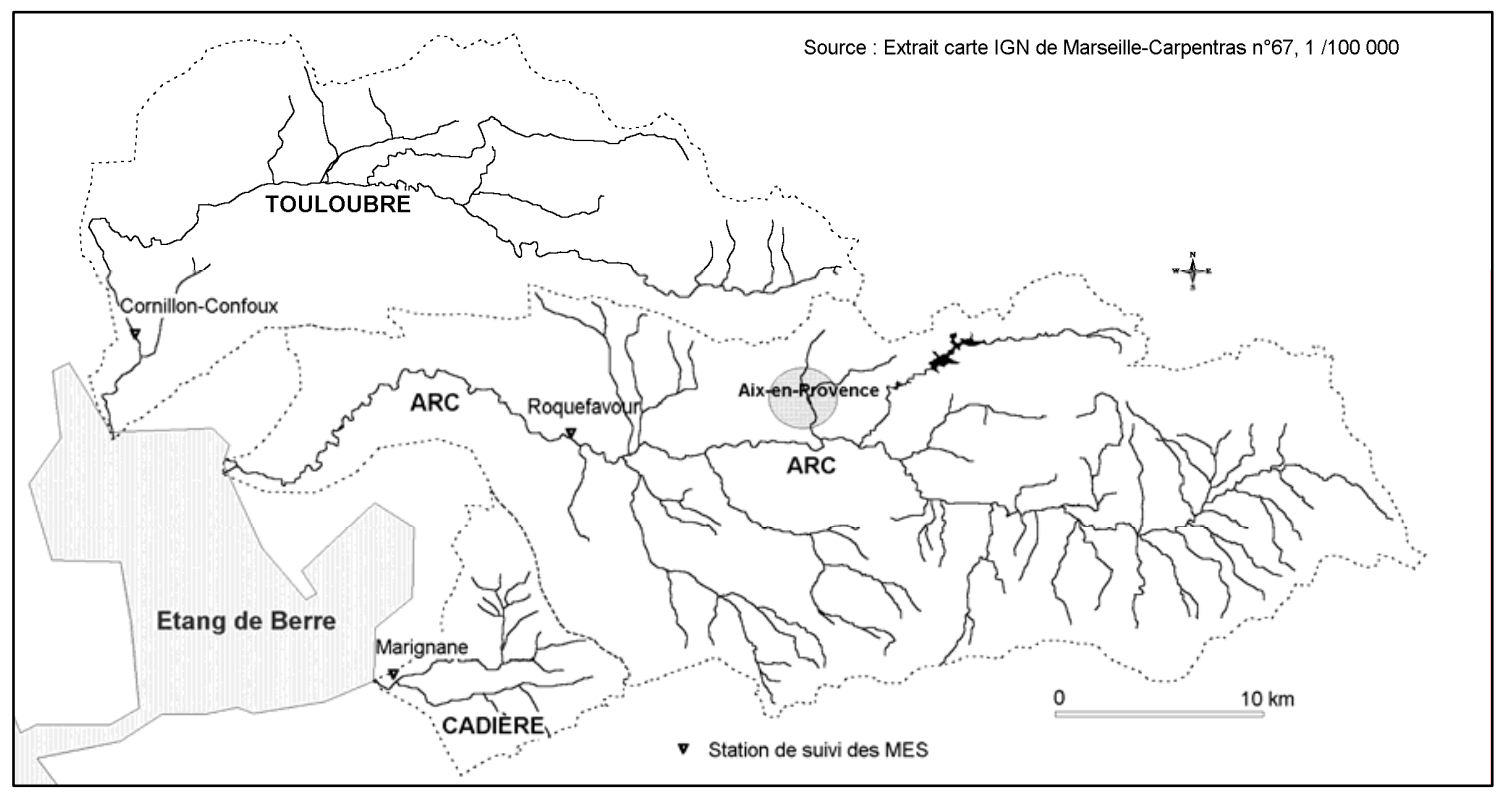

Figure 1 - Présentation du bassin versant de l'Etang de Berre.

Tableau I - Densité de population et occupation du sol dans les bassins versants.

\begin{tabular}{|l|l|l|l|}
\hline & \multicolumn{1}{|c|}{ Arc } & \multicolumn{1}{c|}{ Touloubre } & \multicolumn{1}{c|}{ Cadière } \\
\hline Densité de population & 350 habitants $/ \mathrm{km}^{2}$ & 250 habitants $/ \mathrm{km}^{2}$ & 1400 habitants $/ \mathrm{km}^{2}$ \\
$\begin{array}{l}\text { Occupation du sol } \\
\text { (en \% de la superficie } \\
\text { totale) }\end{array}$ & $\begin{array}{l}\text { Agriculture : 40 } \\
\text { Pinèdes, garrigues : } 50\end{array}$ & $\begin{array}{l}\text { Agriculture : 53 } \\
\text { Pinèdes, garrigues : 42,5 }\end{array}$ & $\begin{array}{l}\text { Agriculture : 25 } \\
\text { Pinèdes, garrigues : } 50 \\
\text { Urbanisation : 25 }\end{array}$ \\
\hline
\end{tabular}

Tableau II - Lois générales de fonctionnement hydrosédimentaire des cours d'eau d'après les conditions hydrologiques.

\begin{tabular}{|c|c|c|}
\hline Bassin & Crues & Faibles écoulements \\
\hline Arc & $\begin{array}{l}\text { Fj = 0,79560 Iq }{ }^{2}+123,09729 \text { Iq - 733,10436 } \\
r=0,95 \text { et }^{2}=0,91, \\
\text { avec Iq = (0,8 Qj + 0,2 Qimax) (DQ/Qimax) }\end{array}$ & $\begin{array}{l}\mathrm{Fj}=9,5315 \mathrm{Qj}-9,0776 \\
\mathrm{r}=0,82 \text { et } \mathrm{r}^{2}=0,68\end{array}$ \\
\hline Touloubre & $\begin{array}{l}\mathrm{Fj}=1,2249 \mathrm{Iq}^{3}-10,574 \mathrm{I} \mathrm{Iq}^{2}+56,074 \mathrm{Iq}-81,957 \\
\mathrm{r}=0,95 \text { et r}^{2}=0,90 \\
\text { avec Iq }=0,7 \mathrm{Qj}+0,3 \text { Qimax }\end{array}$ & $\begin{array}{l}F j=8,2907 \text { Qj }-8,5894 \\
r=0,77 \text { et } r^{2}=0,60\end{array}$ \\
\hline Cadière & $\begin{array}{l}\mathrm{Fj}=0,1795 \mathrm{Iq}^{3}-3,1021 \mathrm{Iq}^{2}+46,7 \mathrm{Iq}-45,313 \\
\mathrm{r}=0,98 \text { et }^{2}=0,97 \\
\text { avec Iq= (0,65 Qj + 0,35 Qimax) (DQ/Qimax) }\end{array}$ & $\begin{array}{l}\mathrm{Fj}=3,9371 \mathrm{Qj} \\
\mathrm{r}=0,91 \text { et } \mathrm{r}^{2}=0,82\end{array}$ \\
\hline
\end{tabular}

Fj : Flux journalier de MES (tonnes). Iq : Indice traduisant l'agressivité érosive journalière potentielle. $\mathrm{r}$ : Coefficient de corrélation. $\mathrm{r}^{2}$ : Coefficient de détermination. Qj : Débit journalier, en $\mathrm{m}^{3} / \mathrm{s}$. Qimax : Débit instantané maximum, en $\mathrm{m}^{3} / \mathrm{s}$. DQ : Différence entre les débits instantanés maximum et minimum, en $\mathrm{m}^{3} / \mathrm{s}$. 
Tableau III - Lois générales de fonctionnement des cours d'eau d'après les conditions pluviométriques.

\begin{tabular}{|l|l|c|c|}
\hline \multicolumn{1}{|c|}{ Bassin } & \multicolumn{1}{|c|}{ Equation } & $\begin{array}{c}\text { Coefficient de } \\
\text { corrélation } r\end{array}$ & $\begin{array}{c}\text { Coefficient de } \\
\text { détermination } \mathrm{r}^{2}\end{array}$ \\
\hline Arc & $\begin{array}{l}\text { F/mois }=0,413 \mathrm{Ip}^{2}+19,78 \mathrm{Ip}, \\
\text { avec Ip }=(0,2 \mathrm{P} 2 \mathrm{jc})+(0,8 \mathrm{P} 4 \mathrm{j})\end{array}$ & 0,80 & 0,64 \\
\hline Touloubre & $\mathrm{F} / \mathrm{mois}=10,284 \mathrm{P} 2 \mathrm{jc}+284,74$ & 0,64 & 0,41 \\
\hline Cadière & $\begin{array}{l}\mathrm{F} / \mathrm{mois}=0,0013 \mathrm{Ip}^{3}-0,1658 \mathrm{Ip}^{2}+12,667 \mathrm{Ip}, \\
\text { avec Ip }=(0,6 \mathrm{P} 2 \mathrm{jc}+0,4 \mathrm{P} 4 \mathrm{j})\end{array}$ & 0,96 & 0,92 \\
\hline
\end{tabular}

F/mois : Flux mensuel de MES, en tonnes. Ip : Indice traduisant l'agressivité érosive mensuelle potentielle. P2jc : Pluies maximales en deux jours consécutifs au cours du mois, en mm. P4j : Pluies maximales en quatre jours non consécutifs au cours du mois, en mm.

Tableau IV - Erosions spécifiques annuelles moyennes sur la période 1998-2000 : comparaison des deux méthodes de calcul.

\begin{tabular}{|l|c|c|c|}
\hline & Arc & Touloubre & Cadière \\
\hline Précipitations annuelles moyennes $(\mathrm{mm})$ & 614 & 582 & 589 \\
\hline Lame d'eau écoulée annuelle moyenne $(\mathrm{mm})$ & 127 & 196 & 340 \\
\hline $\begin{array}{l}\text { Erosion spécifique }\left(\mathrm{t} / \mathrm{km}^{2} / \mathrm{an}\right) \\
\text { d'après les conditions hydrologiques }\end{array}$ & 36,2 & 16,8 & 56,9 \\
\hline $\begin{array}{l}\text { Erosion spécifique }\left(\mathrm{t} / \mathrm{km}^{2} / \mathrm{an}\right) \\
\text { d'après les conditions pluviométriques }\end{array}$ & 38,3 & 17,4 & 56,5 \\
\hline
\end{tabular}

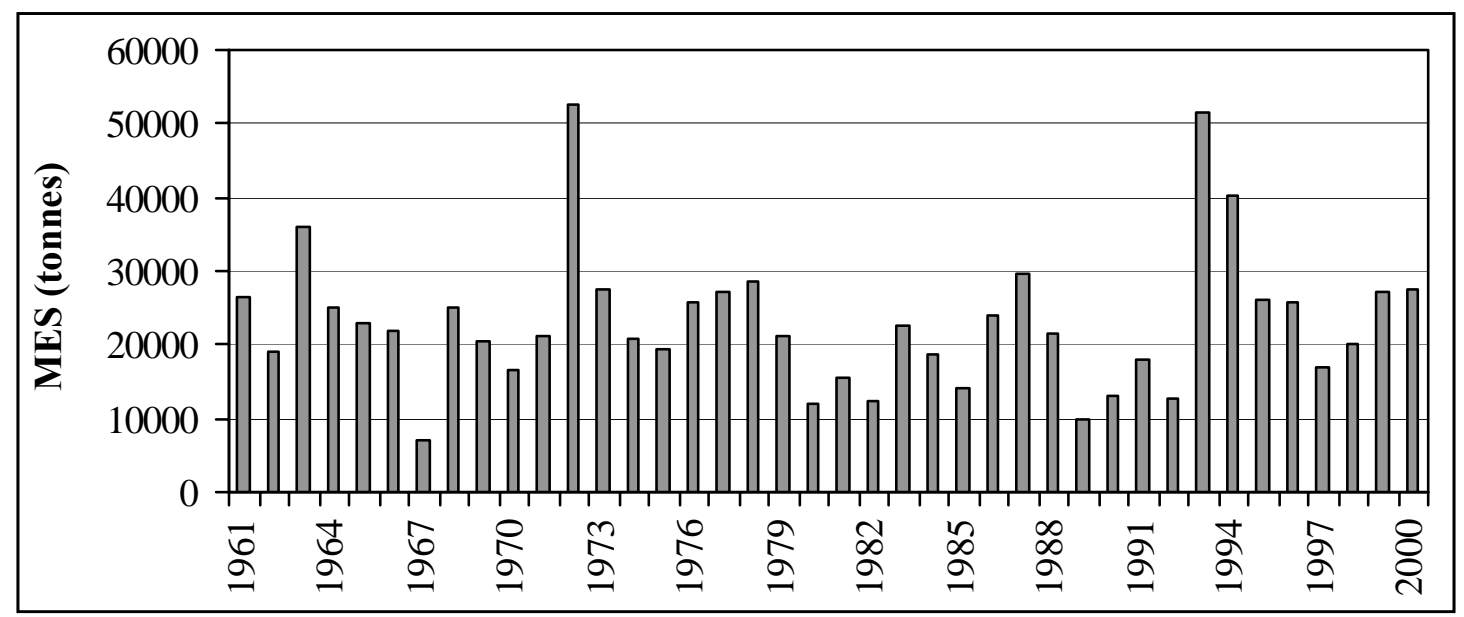

Figure 2 - Exportations annuelles de MES de l'Arc à Roquefavour sur la période 1961-2000. 